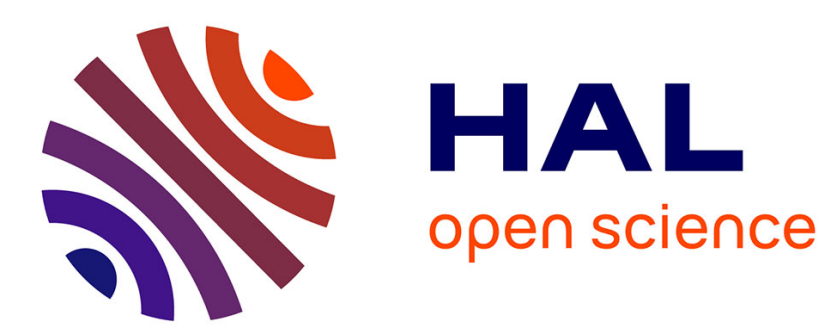

\title{
La mixité des genres dramatiques dans le théâtre de Rotrou
}

\author{
Sandrine Berrégard
}

\section{To cite this version:}

Sandrine Berrégard. La mixité des genres dramatiques dans le théâtre de Rotrou. Colloque "Le théâtre de Rotrou", 2005, Tours, France. pp.97-106. hal-02966374

\section{HAL Id: hal-02966374 \\ https://hal.science/hal-02966374}

Submitted on 13 Oct 2020

HAL is a multi-disciplinary open access archive for the deposit and dissemination of scientific research documents, whether they are published or not. The documents may come from teaching and research institutions in France or abroad, or from public or private research centers.
L'archive ouverte pluridisciplinaire HAL, est destinée au dépôt et à la diffusion de documents scientifiques de niveau recherche, publiés ou non, émanant des établissements d'enseignement et de recherche français ou étrangers, des laboratoires publics ou privés. 


\title{
Sandrine Berregard
}

(Université Marc-Bloch-Strasbourg II),

\section{La mixité des genres dramatiques dans le théâtre de Rotrou}

\author{
Littératures classiques, $\mathrm{n}^{\circ}$ 63: Le Théâtre de Rotrou (actes de colloque), dir. P.Pasquier, 2007, \\ p.97-106
}

La diversité des approches proposées par la critique autour de la question des genres littéraires montre à quel point le sujet est complexe : difficile, en effet, d'établir une classification des textes cohérente $^{1}$, difficile également de déterminer avec certitude les limites d'un genre, difficile enfin d'éviter les anachronismes dans la dénomination des genres. Ainsi, la distinction traditionnellement établie entre poésie, théâtre et roman ${ }^{2}$ n'est guère pertinente en ce qui concerne la littérature française du dix-septième siècle, puisque le théâtre versifié est alors considéré comme faisant intégralement partie du genre poétique au sens large ${ }^{3}$. Pour le définir très sommairement, nous dirons qu'un genre regroupe un ensemble de textes qui présentent des caractéristiques communes, relatives à la forme et au contenu, et qui de ce fait peuvent recevoir la même appellation. L'identité générique d'un texte est établie par l'auteur lui-même ou, à défaut, par le lecteur au moyen d'un rapprochement avec d'autres textes similaires.

Comme l'ont déjà remarqué certains critiques, le sujet est particulièrement difficile à traiter dans le cas de Rotrou ${ }^{4}$. Nombreuses en effet, parmi les trente-cinq pièces qu'il a laissées, sont celles qui procèdent de différents genres et dont le mode de dénomination ne suffit donc pas à refléter toute la complexité. La prédilection que manifeste l'auteur pour la tragi-comédie ${ }^{5}$, elle-même fondée sur le principe de la mixité générique, témoigne ainsi plus largement des orientations esthétiques qui sont les siennes dans l'ensemble de son théâtre. Néanmoins, l'objet de la présente étude n'est pas précisément d'examiner les relations que les pièces de Rotrou font apparaître entre les différents genres dramatiques ${ }^{6}$, mais plutôt de comprendre le rapport qui s'établit entre les modes de dénomination et la réalité des textes. Comment donc expliquer l'écart qui existe parfois entre les deux, que révèlent-ils sur la pratique des genres chez Rotrou, et enfin quel type de relation l'auteur établit-il ainsi avec son

\footnotetext{
${ }^{1}$ J.-M. Schaeffer distingue dans ce domaine trois attitudes possibles : «a/ le paradigme biologique et l'attitude essentialiste qu'il implique ; b/ une attitude descriptive-analytique ; c/ une attitude normative » (Qu'est-ce qu'un genre littéraire?, Paris, Seuil, 1989, p. 13).

${ }^{2}$ Voir, à ce sujet, l'article de G. Genette «Introduction à l'architexte », p. 89-159 dans Théorie des genres, dir. G. Genette et T. Todorov, Paris, Seuil (Points), 1986.

${ }^{3}$ Aussi le théâtre en vers est-il désigné sous le nom de poésie dramatique.

${ }^{4}$ Pour une vue d'ensemble du problème, on se reportera à l'analyse de J. Morel, p. 136-137 dans Jean Rotrou dramaturge de l'ambiguïté, Paris, Colin, 1968.

${ }^{5}$ On compte en effet dix-sept tragi-comédies sur un total de trente-cinq pièces.

${ }^{6}$ Les articles de B. Louvat et de H. Baby, contenus dans le présent volume, traitent plus spécifiquement de cet aspect du sujet.
} 
lecteur? Telles sont les principales questions auxquelles cette étude se propose d'apporter des éléments de réponse.

\section{Modes de dénomination et réalité des textes}

L'œuvre de Rotrou se divise en trois grands ensembles (tragédies, tragi-comédies et comédies), à l'intérieur desquels il est possible de distinguer des sous-ensembles, déterminés par la structure des pièces et/ou par la nature de leurs sujets : tragédies historiques (Crisante, Le Véritable Saint Genest et Cosroès) et tragédies mythologiques (Hercule mourant et Antigone), tragi-comédies de palais (Cléandre ou l'Heureux Naufrage, Agésilan de Colchos, Célie ou le Vice-roi de Naples, Don Bernard de Cabrère et Don Lope de Cardone) et tragi-comédies de la route (L'Hypocondriaque ou le Mort amoureux, La Doristée, L'Heureuse Constance, Les Occasions perdues, L'Innocente Infidélité, Angélique ou la Pèlerine amoureuse, Laure persécutée et Les Deux Pucelles) ${ }^{7}$, et enfin comédies latines (Les Ménechmes, Les Captifs ou les Esclaves et Les Sosies) et comédies à l'italienne (Clarice ou l'Amour constant et $\mathrm{La}$ Sæeur $)^{8}$. Si elle est commode, cette classification exclut néanmoins un assez grand nombre de pièces, dont le statut est plus problématique, soit parce que le genre par lequel elles sont désignées ne rend pas compte de leurs spécificités, soit parce que le mode de dénomination est tout simplement en décalage voire en contradiction (apparente ?) avec la réalité du texte.

L'ambiguïté sur laquelle repose la dramaturgie rotrouesque se traduit aussi par des changements de dénomination, qui affectent précisément certaines de ces pièces : ainsi, Bélisaire est qualifié successivement de tragédie (en page de titre) et de tragi-comédie (entre la liste des personnages et la première scène $)^{9}$; «tragédie » dans l'édition originale de 1641, Iphigénie devient à son tour « tragicomédie » un an plus tard dans la deuxième édition ${ }^{10}$; inversement, Venceslas, présenté à l'origine comme une tragi-comédie, reçoit par la suite le qualificatif de tragédie ${ }^{11}$. Ces fluctuations sont la preuve que les pièces de Rotrou ne répondent que très approximativement aux critères de définition des genres et montrent donc à quel point il est difficile d'établir entre eux des distinctions très nettes.

\footnotetext{
${ }^{7}$ Cette distinction est due à J. Morel, op. cit., p. 169. À l'appellation de «tragi-comédie de palais » H. Baby, quant à elle, préfère celle de «tragi-comédie d'intrigue » (La Tragi-comédie de Corneille à Quinault, Paris, Klincksiek, Bibliothèque de l'âge classique, 2001, p. 143).

${ }^{8}$ L'édition du Théâtre complet de Rotrou, dirigée par G. Forestier, reprend très largement cette typologie. La réunion en un seul volume de La Sæur et de Célie, respectivement comédie et tragi-comédie, se justifie par le fait que les deux pièces sont des adaptations de comédies italiennes (voir, à ce sujet, l'introduction de C. Bourqui, p. 8 dans Théâtre complet, t. 3, Paris, S.T.F.M., 2000), mais elle montre également qu'il est difficile de réduire le théâtre de Rotrou à une classification stricte. De même, comme le remarque C. Bourqui, les comedias espagnoles ont donné lieu tantôt à des comédies ( $\mathrm{La}$ Bague de l'oubli et La Belle Alphrède) tantôt à des tragi-comédies (Laure persécutée et Bélisaire) (ibid.).

${ }^{9}$ Dans son introduction à Bélisaire, M. Béthery remarque ce changement de dénomination (Théâtre complet, t. 1, 1998, p. 20) et définit la pièce comme une tragi-comédie de palais (p. 21 et $s q q$.), tout en la rattachant à l'esthétique de la tragédie (p. 33 et sqq.).

${ }^{10}$ Voir la description que fait A. Riffaud des éditions successives de la pièce (Théâtre complet, t. 2, 1999, p. 398399).

${ }^{11}$ Même si elle présente certaines des caractéristiques propres à la tragédie, Venceslas semble pouvoir être considérée comme une tragi-comédie (voir, à ce sujet, l'introduction de M Béthery, p. 207 et sqq. dans Théatre complet, t. 1).
} 
La difficulté est accrue par le fait que les pièces ont en commun des thèmes, des situations et/ou des types de personnages ${ }^{12}$. Le déguisement, la feinte, l'illusion figurent ainsi parmi les motifs privilégiés par l'auteur dans l'ensemble de son œuvre et constitutifs de sa dramaturgie, sans que toutefois ils puissent être considérés comme caractéristiques d'un genre en particulier ${ }^{13}$. Il semble en effet que Rotrou se situe délibérément à la frontière des genres et joue de l'ambiguïté qui caractérise certains d'entre eux: la tragi-comédie, que l'auteur dans ses dernières pièces (Iphigénie, Bélisaire et Venceslas) tend à confondre avec la tragédie, conformément à l'évolution du genre à partir des années $1640^{14}$, mais aussi et surtout la comédie, qui dès la fin des années 1620 connaît de profondes mutations ${ }^{15}$ et dont le statut générique est de ce fait très incertain ${ }^{16}$.

En outre, étant donné qu'il est impossible de reconstituer dans son intégralité la chronologie des pièces de Rotrou, puisque de nombreuses incertitudes demeurent sur leurs dates de création ${ }^{17}$, il est difficile de retracer très précisément la carrière de l'auteur ${ }^{18}$, même si une lecture globale de son œuvre fait apparaître le souci de répondre précisément aux attentes successives du public contemporain. Ainsi, le choix de la tragi-comédie pour sa première pièce (L'Hypocondriaque, jouée en 1628 à l'Hôtel de Bourgogne) s'explique non seulement par le désir de poursuivre l'entreprise menée par Hardy, auquel Rotrou succéda en tant que poète officiel de la troupe et qui lui-même s'était illustré dans ce genre, mais aussi et surtout par la volonté de participer au développement de la tragi-comédie, qui reçoit encore les faveurs du public, avant que l'intérêt du dramaturge ne se porte vers la tragédie (mythologique puis historique) ${ }^{19}$. De même, il est fort probable que le succès remporté dès la fin des années 1620 par les premières comédies de Corneille, inspirées du modèle pastoral ${ }^{20}$, ait donné au jeune poète l'envie de reprendre la formule.

En un temps où les genres dramatiques entretiennent des relations étroites, la mixité générique correspond à une pratique relativement courante, mais à l'évidence elle se manifeste avec une plus grande acuité chez Rotrou que chez les dramaturges contemporains ${ }^{21}$. L'Illusion comique, qui est

\footnotetext{
${ }^{12}$ On se reportera à l'article que $\mathrm{H}$. Baby, dans le présent volume, consacre à la figure du soldat fanfaron dans les comédies et tragi-comédies de Rotrou.

${ }^{13}$ Ainsi, comme le remarque J. Morel, le thème de la « conquête de la vérité à travers une feinte » est commun aux comédies de Rotrou et à sa tragédie du Véritable saint Genest (op. cit., p. 168).

${ }^{14}$ À propos des relations entre les deux genres, voir H. Baby, op. cit., p. 76 et sqq.

${ }^{15}$ Comme il l'explique dans l'examen de Mélite, Corneille entreprend avec cette première pièce de créer un nouveau type de comédie, qui s'éloigne des conventions héritées de la farce (Euvres complètes, t. 1, éd. de G. Couton, Paris, Gallimard, Pléiade, 1980, p. 5-6).

${ }^{16}$ Voir le développement que G. Conesa consacre au sujet dans La Comédie de l'âge classique (1630-1715), Paris, Seuil (Écrivains de toujours), 1995, p. 37-39.

${ }^{17}$ Voir à ce sujet J. Lelièvre, «Pour une chronologie de Rotrou », p. 255-258 dans Revue d'histoire du théâtre, III, 1950.

${ }^{18}$ Ainsi, on sait que sa première pièce, L'Hypocondriaque, fut représentée en 1628, que la deuxième, La Bague de l'oubli, le fut en 1629 et Hercule mourant en 1634.

${ }^{19}$ Hercule mourant et Antigone sont publiés respectivement en 1636 et 1639, Crisante, Le Véritable saint Genest et Cosroès en 1640, 1647 et 1649.

${ }^{20}$ À propos de Mélite, représentée en 1629, Corneille dit que « le succès en fut surprenant » (éd. cit., p. 5).

${ }^{21}$ Ainsi, comme l'explique G. Forestier à propos de Mélite, Corneille, en introduisant le thème de la fausse mort, caractéristique de la tragi-comédie, se situe à la limite du genre comique ( Corneille et les pratiques d'écriture ", à paraître dans Pratique(s) de Corneille, actes du colloque de Rouen (6-9 juin 2006), dir. M. Maître, Rouen, P.U.R.H.).
} 
fondée sur le principe de la diversité, comme l'illustre l'expression d'« étrange monstre ${ }^{22}$ par laquelle l'auteur lui-même la qualifie, constitue ainsi une exception dans l'œuvre de Corneille. Quant aux changements de dénomination qui affectent certaines de ses pièces (le Clitandre, par exemple, rebaptisé tragédie dans l'édition des Euvres complètes en 1660), ils s'accompagnent d'une réécriture du texte ${ }^{23}$ et participent d'une stratégie, dont la principale visée est de donner à l'ensemble du théâtre cornélien une véritable cohérence générique. Chez Rotrou, la concentration de thèmes et/ou l'utilisation massive de procédés propres à un genre dans une pièce que son mode de dénomination rattache à un autre genre conduit à s'interroger sur son identité générique réelle et sur le rôle de l'auteur et/ou de l'éditeur dans ce processus. Ainsi, que recouvre précisément le mot comédie, par lequel Rotrou désigne un ensemble de pièces très disparate ? Ou encore, la forte présence du modèle pastoral dans son œuvre ${ }^{24}$ suffit-elle à expliquer l'absence de pièces appelées ainsi chez un auteur qui, tout au long de sa carrière, manifesta son goût pour l'expérimentation, et alors même qu'il existe au dix-septième siècle des qualificatifs susceptibles de s'appliquer à certaines de ses pièces (« tragicomédie pastorale $», ~ «$ comédie pastorale $\left.»^{25}\right)$ ?

\section{L’exemple de « comédies pastorales »}

Le cas sans doute le plus problématique, mais aussi le plus révélateur en ce qui concerne Rotrou et sa pratique des genres, est celui des " comédies pastorales »-selon un mode de dénomination qui s'est récemment imposé dans les études consacrées à 1 'auteur ${ }^{26}$. C'est en effet ainsi que J. Morel et C. Scherer désignent La Céliane, Diane, La Célimène, Le Filandre, La Clorinde et La Florimonde, dont la structure (la chaîne des amours non-partagées) et aussi parfois le décor (un cadre champêtre) sont empruntés au modèle pastoral ${ }^{27}$. L'histoire de l'Amarillis, la pastorale dramatique créée par Tristan à partir du brouillon qui avait permis à Rotrou de composer une comédie (La Célimène $)^{28}$, montre

\footnotetext{
22 «À Madamoiselle M.F.D.R.», p. 613 dans CEuvres complètes, éd. cit., t. 1. Et Corneille d'ajouter : «Le premier Acte n'est qu'un Prologue, les trois suivants font une Comédie imparfaite, le dernier est une Tragédie, et tout cela cousu ensemble fait une Comédie ».

${ }^{23}$ Corneille supprime ainsi plus de deux cents vers (voir, à ce sujet, la notice de G. Couton, ibid., p. 1199-1200). De «tragi-comédie » Le Cid devient «tragédie» dans l'édition des Euvres complètes en 1648; G. Couton explique ce changement par la désaffection du public à l'égard du genre tragi-comique dès cette époque-là (ibid., p. 1466).

${ }^{24}$ Voir, à ce sujet, J. Morel, op. cit., p. 137 et sqq., 164 et sqq.

${ }^{25}$ Ainsi, l'atmosphère de galanterie, qui distingue la tragi-comédie d'Amélie, conduit $\mathrm{H}$. Baby à la qualifier de « tragi-comédie pastorale » (Théâtre complet, éd. cit., t. 5, 2002, p. 264).

${ }^{26}$ Alors que la mixité générique, qui caractérise ces pièces, suscite l'intérêt de la critique actuelle, aux dixhuitième et dix-neuvième siècles elle est perçue comme une maladresse. Héritier de cette représentation, J. Marsan, dans l'ouvrage qu'il consacre à la pastorale dramatique, juge ainsi avec sévérité la comédie pastorale telle que l'illustre la Mélite de Corneille : "Cette comédie vient d'éclore, qui, lentement, se préparait dans le fatras des productions médiocres, confuses et surchargées de la pastorale » (J. Marsan, La Pastorale dramatique en France à la fin du XVI et au commencement du XVII siècle, Paris, [s.n.], 1905, p. 361).

${ }^{27}$ C. Scherer, Comédie et société sous Louis XIII. Corneille, Rotrou et les autres, Paris, Nizet, 1983, p. 57, 66, etc. ; J. Morel, op. cit., p. 165.

${ }^{28}$ Voir, à ce sujet, l'édition critique de l'Amarillis réalisée par R. Guichemerre (Euvres complètes, t. 5, Paris, Champion, Sources classiques, 1999, p. 119), et l'édition critique de La Célimène réalisée par V. Lochert (Théâtre complet, éd. cit., t. 6, 2003, p. 13-14).
} 
en effet que les deux genres sont dans un rapport de proximité étroite. Néanmoins, à la dénomination de « comédies pastorales » J. Morel en adjoint une autre, celle de "pastorales pures »-expression pour le moins paradoxale au regard de la mixité générique qui caractérise ces pièces - en expliquant qu'il qualifie ainsi des œuvres dramatiques « de structure fermée, dont le style s'apparente tour à tour à la manière lyrique et à la manière élégiaque, et dont l'action, qui exclut l'aventure et les surprises non préparées, se déroule selon les exigences d'une certaine logique, voire d'une certaine géométrie $»^{29}$. De cet ensemble il exclut $L a$ Clorinde pour ses « aspects franchement comiques », liés pour l'essentiel au personnage de Polydor, sorte de soldat fanfaron ${ }^{30}$. Le caractère approximatif de la terminologie (« pastorales pures », « comédies de couleur pastorale », « comédies de type pastoral » ${ }^{31}$, etc.), ajouté à la présence dans cette série d'une pièce que l'édition originale présente comme une tragi-comédie (La Céliane) ${ }^{32}$, témoigne une nouvelle fois de la difficulté à caractériser les œuvres en termes génériques, comme si le théâtre de Rotrou se situait hors des cadres traditionnels. À cette difficulté s'en ajoute encore une autre, relative aux diverses interprétations qui peuvent être faites de la pastorale, considérée tantôt comme un genre à part entière, capable de donner son nom à des pièces ${ }^{33}$, tantôt comme une catégorie esthétique, c'est-à-dire comme un ensemble de thèmes et d'images susceptibles de s'incarner dans différents genres ${ }^{34}$. La question est donc de savoir si les motifs empruntés à la tradition pastorale constituent de simples ornements ou si, au contraire, leur présence suffit à remettre en cause l'appartenance des pièces de Rotrou au genre de la comédie.

Les textes théoriques que le dix-septième siècle consacre au sujet ne permettent guère de résoudre les difficultés, dans la mesure où ils ne prennent pas en considération tous les cas de mixité générique. Ainsi, pour distinguer les trois genres que sont la tragédie, la comédie et la pastorale, d'Aubignac, dans sa Pratique du théâtre, mentionne les décors et les types de personnages qui leur sont respectivement liés : «Comme il y a trois sortes de Vies, celle des Grands dans la Cour des Rois, celle des Bourgeois dans les Villes, et celle des gens de la Campagne ; le Théâtre aussi a reçu trois genres de Poèmes Dramatiques qui portent en particulier le caractère de chacune de ces trois sortes de Vies, savoir la Tragédie, la Comédie, et la Satyre ou Pastorale » ${ }^{35}$. Dès lors, comment situer une pièce comme La Florimonde, qui, par son décor bucolique, issu de L'Astrée, relève du genre pastoral, mais dont les personnages, venus de la ville, appartiennent à la bourgeoisie ${ }^{36}$ ?

\footnotetext{
${ }^{29}$ J. Morel, op. cit., p. 137.

${ }^{30}$ Ibid., p. 142.

${ }^{31}$ Ibid., p. 109 et 168.

${ }^{32}$ La Céliane, Paris, Quinet, 1637.

${ }^{33}$ Comme Les Bergeries de Racan (1625) et L'Amaranthe de Gombauld (1631).

${ }^{34}$ Voir, à ce sujet, l'article de D. Dalla Valle consacré à la pastorale dans Dictionnaire des genres et notions littéraires, nouvelle édition augmentée, Paris, A. Michel, $2^{\mathrm{e}}$ éd., Encyclopedia Universalis, 2001, p. 565.

${ }^{35}$ La Pratique du théâtre, éd. critique de H. Baby, Paris, Champion (Lumières classiques), 2001, livre second, chap. 10, p. 209. On se reportera également au développement que l'auteur consacre, dans ce même chapitre, à la « Satyrique » ou « Pastorale », fondée sur le « mélange de choses sérieuses et bouffonnes » (p. 214-215).

${ }^{36}$ Félicie, sous le nom de Tyrsis, indique qu'elle est venue en « Forez » pour se venger de son amant infidèle (III, 8, v. 941), mais elle précise également que «Lyon » est sa ville d'origine (v. 900). En outre, le raffinement qui caractérise les personnages laisse à penser qu'ils appartiennent à la bourgeoisie, au même titre que les jeunes gens mis en scène par Corneille dans ses premières pièces.
} 
Malgré les évidentes analogies qu'elle présente avec les autres, chacune des comédies pastorales imaginées par Rotrou possède des caractéristiques qui lui sont propres, et la question de son rapport $\mathrm{au}(\mathrm{x})$ genre(s) se pose de manière spécifique ${ }^{37}$. Il semble donc que l'auteur use des diverses possibilités qui lui sont offertes à l'intérieur d'un seul et même cadre, empêchant ainsi le lecteur d'établir des règles précises communes à l'ensemble du corpus. Nous prendrons, à titre d'exemples, La Clorinde et La Florimonde, qui méritent d'être retenues pour leurs particularités respectives. La première s'apparente au modèle cornélien de la comédie urbaine ${ }^{38}$, inauguré par Mélite en $1629^{39}$, sans toutefois s'y conformer jusqu'au bout, puisque, contrairement aux quatre premiers, le dernier acte se situe dans un cadre bucolique ${ }^{40}$, à l'exemple de La Céliane, de La Célimène et de La Florimonde, et multiple les péripéties propres à la tragi-comédie (ainsi, l'héroïne se déguise en guerrier pour reconquérir le cœur de son amant) ${ }^{41}$. En outre, la présence du soldat fanfaron permet à l'auteur de $L a$ Clorinde de renouer avec la tradition du genre comique, que Corneille avait préféré tenir à distance, afin de donner à la comédie ses lettres de noblesse ${ }^{42}$. Toutefois, l'incursion dans les domaines de la pastorale et de la tragi-comédie ne suffit pas à remettre en cause l'appartenance effective de $L a$ Clorinde au genre comique, étant donné que les motifs qui s'y rattachent jouent dans la construction de la pièce un rôle secondaire et que leur utilisation n'était pas nécessaire au dénouement de l'intrigue (même si l'atmosphère créée par le décor pastoral favorise la réconciliation des amoureux, cet événement aurait pu survenir dans le décor originel ${ }^{43}$; mais en revanche, elle montre que Rotrou, à la différence d'un Corneille par exemple, situe volontiers la comédie au carrefour des genres, y compris dans une pièce qui aurait pu appartenir à une seule catégorie générique. Ainsi, l'inscription dans le décor du Forez, les costumes de bergers, que portent vraisemblablement les personnages, invitent le lecteur à considérer La Florimonde, sinon comme une véritable pastorale ${ }^{44}$, du moins comme une tragi-comédie pastorale, à l'exemple de certaines œuvres adaptées de L'Astrée $e^{45}$, puisqu'elle accorde une large place à des thèmes spécifiquement attachés au genre tragi-comique (le combat, le voyage de

\footnotetext{
${ }^{37}$ On se reportera aux développements que V. Lochert et L. Picciola consacrent respectivement au sujet dans leurs éditions de La Célimène et de Diane (Théatre complet, éd. cit., t. 6, p. 26 et sqq., 213 et sqq.).

${ }^{38}$ Dans notre édition critique, nous établissons ainsi de nombreux rapprochements entre La Clorinde et les deux comédies de Corneille Mélite et La Galerie du palais (Théâtre complet, t. 9, 2007).

${ }^{39}$ Mélite semble en effet avoir été créée durant la saison 1629-1630 (voir, à ce sujet, la notice de G. Couton, éd. cit., p. 1143).

${ }^{40}$ La scène $1^{\text {re }}$ de l'acte $\mathrm{V}$ précise que Céliandre et Clarimond sont « aux champs ».

${ }^{41}$ La Clorinde, $\mathrm{V}$, scène dernière.

${ }^{42}$ « On n'avait jamais vu jusque-là que la Comédie fît rire sans Personnages ridicules, tels que les Valets bouffons, les Parasites, les Capitans, les Docteurs, etc. »(Corneille, examen de Mélite, p. 6 dans éd. cit.).

${ }^{43}$ Aussi sommes-nous en désaccord avec R. Guichemerre, qui estime que «les péripéties romanesques » de $L a$ Clorinde « en font plutôt [une tragi-comédie] » (La Comédie avant Molière 1640-1660, Paris, A. Colin, 1972, p. 15).

${ }^{44}$ Aussi l'affirmation de J. Morel selon laquelle Diane, La Célimène, Le Filandre et La Florimonde sont « de véritables pastorales » n'est-elle guère en accord avec la réalité des textes (op. cit., p. 142).

${ }^{45}$ Parmi ces pièces figurent la Tragi-comédie pastorale, où les Amours d'Astrée et de Céladon sont mêlées à celles de Diane de Rayssiguier, La Clenide de La Barre et L'Inconstance d'Hylas de Mareschal, publiées respectivement en 1630,1634 et 1635 .
} 
reconquête et la fausse mort) ${ }^{46}$. Néanmoins, l'appartenance de l'intrigue principale à la comédie d'intrigue (Florimonde feint d'être amoureuse de Théaste, afin d'éveiller la jalousie de Cléante, indifférent à son égard) empêche de réduire la pièce aux genres de la pastorale et de la tragi-comédie, et montre ainsi que la comédie est la seule des trois à pouvoir transcender les autres catégories génériques.

\section{Une négligence délibérée}

Il semble en effet que Rotrou joue de l'ambiguïté sémantique inhérente au mot lui-même dans la langue du dix-septième siècle ${ }^{47}$. Les scènes de divertissement, où le jeu se mêle au rire, invitent certes à considérer la comédie au sens étroit, et le procédé de mise en abyme sur lequel elles reposent permet d'y voir une représentation du dispositif théâtral lui-même ${ }^{48}$ : hypothèse que la présence de personnages franchement comiques, comme le soldat fanfaron dans La Clorinde ou La Belle Alphrède, est de nature à corroborer. Mais le mot peut aussi être entendu en un sens beaucoup plus large et désigner n'importe quelle pièce de théâtre, suivant une acception qu'attestent les dictionnaires de l'époque - à moins que, plus vraisemblablement, il ne corresponde au genre moyen tel que Corneille l'a défini dans l'examen de Mélite $^{49}$ et dont le principal critère discriminant est de s'opposer à la tragédie. La liberté de Rotrou dans sa pratique du genre est d'autant plus grande que jamais l'auteur ne définit la comédie en termes théoriques, et que la seule fois où il prononce le mot pour exprimer un jugement (« Puisque enfin la comédie est en un point où les plus honnêtes récréations ne lui peuvent plus causer d'envie, où elle se peut vanter d'être la passion de toute la France, et le divertissement même de Votre Majes té ... »), on ne sait s'il parle du théâtre en général ou du genre comique en particulier ${ }^{50}$. En regroupant sous une même bannière un ensemble de pièces très hétérogène, l'auteur reconnait donc implicitement le caractère polymorphe du genre.

\footnotetext{
${ }^{46}$ Cet aspect de la pièce sera plus longuement traité dans notre édition critique (Théâtre complet, éd. cit., t. 10, 2008).

${ }^{47}$ Le Dictionnaire de l'Académie française (1694) définit ainsi le mot comédie : «Pièce dramatique, pièce de théâtre représentant quelque action de la vie humaine qui se passe entre des personnes privées. Il se prend plus généralement pour toutes sortes de pièces de théâtre comme sont la tragédie, la tragi-comédie et la pastorale ». Après avoir défini la comédie comme une "pièce de théâtre composée avec art, en prose, ou en vers », le Dictionnaire de Furetière (1690) en donne une autre acception, qui pourrait aussi s'appliquer à la pastorale et à la tragi-comédie ( Se prend plus particulièrement pour les pièces qui représentent des choses agréables et non sanglantes, et des personnes de médiocre condition »), pour rappeler in fine le lien qui depuis ses origines unit la comédie à la tradition farcesque («Se dit encore en un sens plus étroit, pour une farce, une facétie, où on n'introduit guère que des valets et des bouffons, pour dire des choses plaisantes, et faire rire »).

${ }^{48}$ Voir, à ce sujet, l'analyse de V. Lochert dans son édition critique de La Célimène (éd. cit., p. 43 et sqq.).

${ }^{49}$ " La nouveauté de ce genre de Comédie, dont il n'y a point d'exemple en aucune Langue, et le style naïf, qui faisait une peinture de la conversation des honnêtes gens, furent sans doute cause de ce bonheur surprenant, qui fit alors tant de bruit. [...] Celle-ci faisait son effet par l'humeur enjouée de gens d'une condition au-dessus de ceux qu'on voit dans les Comédies de Plaute et de Térence, qui n'étaient que des Marchands » (éd. cit., p. 6-7).

${ }^{50} \mathrm{C}$ 'est par ces mots que s'ouvre l'épître dédicatoire de La Bague de l'oubli, adressée au roi de France. Voir la note qui accompagne cette phrase dans l'édition critique de N. Courtès (Théâtre complet, éd. cit., t. 9).
} 
Reste à savoir si le choix du mode de désignation procède de la volonté du poète lui-même ou relève de l'initiative du (seul ?) libraire, et sur quels principes il est fondé (résulte-t-il, par exemple, d'une stratégie destinée à faire vendre une pièce, à un moment où le genre reçoit les faveurs du public, et alors même que son incertitude générique permettrait de la nommer autrement ?). Le faible intérêt que Rotrou, contrairement à d'autres dramaturges de son temps, portait à l'impression de ses pièces, la distance qui le séparait des ateliers parisiens, à chaque fois qu'il séjournait dans sa ville natale ${ }^{51}$, expliquent sans doute pour une large part l'incertitude qui entoure les modes de dénomination. Mais il est également permis de se demander si Rotrou lui-même n'entretient pas délibérément l'ambiguïté, ou du moins ne s'en accommode pas, dans la mesure où elle lui permet de ne pas révéler immédiatement au lecteur la nature réelle de son texte. À l'exemple de ses nombreux personnages qui dissimulent leur véritable identité en se jouant de leurs interlocuteurs, et dont l'attitude est fondée sur le principe de l'ambiguïté (générique ${ }^{52}$, Rotrou se plaît à surprendre son lecteur en ne se conformant pas toujours à l'horizon d'attente générique créé par le mode de désignation ${ }^{53}$ : ainsi, en contradiction avec les conventions de la tragi-comédie, Bélisaire développe une intrigue inspirée d'un épisode de l'histoire ${ }^{54}$. L'incertitude sémantique qui entoure le mot comédie est donc parfaitement en accord avec l'esthétique de la diversité sur laquelle reposent les « comédies pastorales » de Rotrou : capable de réaliser une sorte de synthèse entre la comédie farcesque, la tragi-comédie et la pastorale, la comédie représente pour l'auteur un extraordinaire espace de liberté et d'invention. Telle est la singularité du poète dans le paysage théâtral de son temps.

Il semble donc que, contrairement à la plupart des dramaturges contemporains, Rotrou ne sépare jamais complètement les différents genres qu'il pratique - d'où l'utilisation d'une terminologie qui révèle l'incertitude générique de ses pièces. Sans être aléatoires-encore qu'on ne puisse définitivement écarter cette hypothèse, à propos de La Belle Alphrède, par exemple, qui présente les caractéristiques d'une tragi-comédie, tout en portant le qualificatif de comédie ${ }^{55}-$ les modes de dénomination témoignent de l'écart qui existe entre les catégories élaborées par les critiques et/ou les théoriciens du temps et les réalités textuelles. Qu'il ne soit finalement pas possible d'établir une classification des textes complète et rigoureuse ne doit donc pas être perçu comme un constat d'échec,

\footnotetext{
${ }^{51}$ On se reportera à l'article qu'A. Riffaud consacre au sujet dans le présent volume.

${ }^{52}$ Parmi ces personnages figurent Céliane (dans la pièce du même nom), Célimène (dans la pièce homonyme) et Félicie (dans La Florimonde).

${ }^{53}$ À propos de ce concept, voir H.R. Jauss, «Littérature médiévale et théorie des genres », p. 41 dans Théorie des genres, op. cit.

${ }^{54}$ Voir, à ce sujet, l'introduction de M. Béthery, éd. cit., p. 13 et sqq.

${ }^{55}$ Aussi l'expression de « comédie romanesque », par laquelle J.-C. Vuillemin qualifie La Belle Alphrède dans son édition critique, peut-elle être considérée comme une autre manière de définir la tragi-comédie (Théatre complet, éd. cit., t. 9).
} 
mais au contraire être compris comme l'expression d'une volonté, celle de donner aux catégories génériques une extension maximale. Ainsi, placée à la jonction de la comédie (entendue au sens étroit), de la pastorale et de la tragi-comédie, la comédie rotrouesque se donne pour but de répondre à l'exigence de plaisir en construisant un imaginaire qui, nourri des lieux communs attachés à ces différents genres, éloigne le spectateur du monde réel ${ }^{56}$.

\footnotetext{
${ }^{56}$ C'est ainsi que C. Scherer oppose à la " dramaturgie du quotidien », incarnée par les premières comédies de Corneille, la «dramaturgie de l'imaginaire », représentée par les comédies pastorales de Rotrou (op. cit., p. 55, 181, etc.).
} 